\title{
Network analysis of differentially expressed smoking-associated mRNAs, IncRNAs and miRNAs reveals key regulators in smoking-associated lung cancer
}

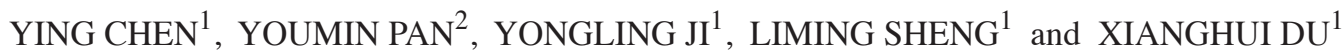 \\ ${ }^{1}$ Department of Radiation Oncology, Zhejiang Key Laboratory of Radiation Oncology, Zhejiang Cancer Hospital; \\ ${ }^{2}$ Department of Blood Transfusion, The First Affiliated Hospital, College of Medicine, \\ Zhejiang University, Hangzhou, Zhejiang 310000, P.R. China \\ Received February 8, 2018; Accepted August 31, 2018
}

DOI: $10.3892 /$ etm.2018.6891

\begin{abstract}
Lung cancer is the leading cause of cancer-associated mortality worldwide. Smoking is one of the most significant etiological contributors to lung cancer development. However, the molecular mechanisms underlying smoking-induced induction and progression of lung cancer have remained to be fully elucidated. Furthermore, long non-coding RNAs (lncRNAs) are increasingly recognized to have important roles in diverse biological processes. The present study focused on identifying differentially expressed mRNAs, lncRNAs and micro (mi)RNAs in smoking-associated lung cancer. Smoking-associated co-expression networks and protein-protein interaction (PPI) networks were constructed to identify hub lncRNAs and genes in smoking-associated lung cancer. Furthermore, Gene Ontology and Kyoto Encyclopedia of Genes and Genomes analyses of differentially expressed lncRNAs were performed. A total of 314 mRNAs, 24 lncRNAs and 4 miRNAs were identified to be deregulated in smoking-associated lung cancer. PPI network analysis identified 20 hub genes in smoking-associated lung cancer, including dynein axonemal heavy chain 7, dynein cytoplasmic 2 heavy chain 1, WD repeat domain 78, collagen type III $\alpha$ 1 chain (COL3A1), COL1A1 and COL1A2. Furthermore, co-expression network analysis indicated that relaxin family peptide receptor 1 , receptor activity modifying protein 2-antisense RNA 1, long intergenic non-protein coding RNA 312 (LINC00312) and LINC00472 were key lncRNAs in smoking-associated lung cancer. A bioinformatics analysis indicated these smoking-associated lncRNAs have a role in
\end{abstract}

Correspondence to: Dr Xianghui Du, Department of Radiation Oncology, Zhejiang Key Laboratory of Radiation Oncology, Zhejiang Cancer Hospital, 1 Banshandong Road, Gongshu, Hangzhou, Zhejiang 310000, P.R. China

E-mail: duxh@zjcc.org.cn

Key words: smoking, long non-coding RNA, lung cancer, miRNAs, protein-protein interaction analysis, co-expression networks various processes and pathways, including cell proliferation and the cyclic guanosine monophosphate cGMP)/protein kinase cGMP-dependent 1 signaling pathway. Of note, these hub genes and lncRNAs were identified to be associated with the prognosis of lung cancer patients. In conclusion, the present study provides useful information for further exploring the diagnostic and prognostic value of the potential candidate biomarkers, as well as their utility as drug targets for smoking-associated lung cancer.

\section{Introduction}

Lung cancer is the leading cause of cancer-associated mortalities worldwide. Non-small cell lung cancer (NSCLC), which constitutes $80 \%$ of lung cancer cases, and SCLC are two major types $(1,2)$. Lung cancer is a highly heterogeneous disease and a large variety of factors are involved in its genesis and progression (3). Bryant and Cerfolio (4) have reported that smoking is one of the most significant etiological factors that contribute to lung cancer development and is associated with $90 \%$ of lung cancer cases. Furthermore, several genes have been indicated to have a role in smoking-associated lung cancer progression. For instance, Lv et al (5) reported that RBM5 inhibits the proliferation of cigarette smoke-transformed BEAS-2B cells through causing cell cycle arrest and apoptosis. Furthermore, polymorphisms of CYPIA1 were indicated to be linked with the risk of smoking-associated lung cancer risk in an Egyptian population (6). However, the molecular mechanisms underlying the smoking-associated genesis and progression of lung cancer have remained largely elusive.

Long non-coding RNAs (lncRNAs) are a class of ncRNAs of $>200$ nucleotides in length and no protein-coding function (7). They have become a novel focus of biological research, as they have been indicated to be important regulators in various diseases by affecting a vast variety of biological processes, including cell cycle, apoptosis and differentiation (8). In lung cancer, lncRNAs have been indicated to have an essential role in the regulation of gene expression at the epigenetic, transcriptional and post-transcriptional levels (9). For instance, IncRNA HOXA distal transcript antisense RNA has been reported to promote B-cell lymphoma-2 expression 
and induce chemoresistance in SCLC by sponging microRNA (miR)-216a (10). In addition, lncRNA LINK-A interacts with Phosphatidylinositol-3,4,5-trisphosphate $[\operatorname{PtdIns}(3,4,5) \mathrm{P} 3$ or PIP3] to hyperactivate AKT and confer resistance to AKT inhibitors (11). However, apart from metastasis associated lung adenocarcinoma (LUAD) transcript 1 (MALAT-1), colon cancer associated transcript 1 (CCAT-1) and long intergenic non-coding RNA 94 (LINC00094), a limited number of lncRNAs were identified to be associated with smoking-induced lung cancer (12). Therefore, identification of lncRNAs with a role in smoking-associated lung cancer may provide novel insight to reveal mechanisms underlying the smoking-induced genesis and progression of the malignancy.

In the present study, the public dataset GSE43458 was analyzed to identify differentially expressed lncRNAs and mRNAs in smoking-associated lung cancer. Next, protein-protein interaction (PPI) and co-expression networks were constructed to identified hub mRNAs and lncRNAs in smoking-associated lung cancer. Furthermore, gene ontology (GO) and Kyoto Encyclopedia of Genes and Genomes (KEGG) pathway analyses were performed to explore the potential roles of the differently expressed genes (DEGs). The present study provides useful information to explore potential candidate biomarkers for diagnosis, prognostication and drug targets for smoking-associated lung cancer.

\section{Materials and methods}

Retrieval and pre-processing of microarray data. The raw dataset GSE43458 (13) was downloaded from the gene expression omnibus (GEO) website (https://www.ncbi.nlm. nih.gov/geo/) and pre-processed by $\log 2$ transformation. A total of 110 samples were included in GSE43458, which included 30 normal samples, 40 NSCLC tissues from never-smoking patients and 40 NSCLC tissues from smoking patients. Furthermore, The Cancer Genome Atlas (TCGA) (https://cancergenome.nih.gov/) LUAD dataset was analyzed to identify smoking-associated miRNAs, including 46 normal samples, 64 LUAD tissues from never-smoking patients and 372 LUAD tissues from smoking patients. All sample data were normalized using the linear models for microarray analysis (limma) package in $\mathrm{R}$ version 3.3.0 (https://www.r-project.org/). The differentially expressed mRNA and lncRNAs were identified by the limma method. The DEGs were obtained with thresholds of llog fold change (FC) $>1.5$ and $\mathrm{P}<0.001$.

The hierarchical cluster analysis of differentially expressed mRNAs and lncRNAs was performed using CLUSTER 3.0 (https://www.geo.vu.nl/ huik/cluster.htm), and the hierarchical clustering heat map was visualized by Tree View (14).

GO and KEGG pathway analysis. To identify functions of DEGs in smoking-associated lung cancer, GO functional enrichment analysis was performed in the categories biological process, cellular component and molecular function. KEGG pathway enrichment analysis was also performed to identify pathways enriched in smoking-associated lung cancer using the Database for the Annotation, Visualization and Integrated Discovery (DAVID; https://david.ncifcrf.gov/). $\mathrm{P}<0.05$ was considered to indicate a statistically significant difference.
IncRNA classification pipeline. In the present study, differently expressed lncRNAs in lung cancer were identified by adopting the criteria reported by Yang et al (15). In brief, first, the GPL570 platform of the Affymetrix Human Genome U133 Plus 2.0 Array (Affymetrix Inc., Santa Clara, CA, USA) probe set ID was mapped to the NetAffx Annotation Files (HG-U133 Plus 2.0 Annotations, CSV format, release 31, 08/23/10). The annotations included the probe set ID, gene symbol and Refseq transcript ID. Subsequently, the probe sets that were assigned a Refseq transcript ID in the NetAffx annotations were extracted. In the present study, only those labeled as 'NR_', indicating non-coding RNA in the Refseq database, were retained. Finally, 2,448 annotated lncRNA transcripts with corresponding Affymetrix probe IDs were generated. IncRNAs with $\mathrm{FC} \geq 2$ and $\mathrm{P}<0.05$ were considered to be significantly differentially expressed.

Construction of PPI network and module analysis. In order to predict protein interactions, including physical and functional associations, the present study used the Search Tool for the Retrieval of Interacting Genes (STRING) to construct the PPI network for DEGs (16). The interaction associations of the proteins encoded by the DEGs were identified using STRING online software, and a combined interaction score of $>0.4$ was used as the cut-off criterion. In addition, Cytoscape software version 3.4.0 (http://www.cytoscape.org/) was used for visualization of the PPI networks (17). Following the construction of the PPI network, a module analysis of the network was performed using the Mcode plugin (degree cut-off, $\geq 2$; nodes with edges, $\geq 2$-core) (18). In addition, the Network Analyzer was used to compute the basic properties of the PPI network, including average clustering coefficient distribution, closeness centrality, average neighborhood connectivity, node degree distribution, shortest path length distribution and topological coefficients (19).

Co-expression network construction and analysis. In the present study, the Pearson correlation coefficients of differently expressed mRNA-lncRNA pairs were calculated according to their expression value. The co-expressed DEG-IncRNA pairs with Pearson correlation coefficients with an absolute value of $\geq 0.75$ were selected and the co-expression network was generated by using Cytoscape software. The Cytoscape Mcode plug-in was applied for visualization of the co-expression networks.

Survival analysis. Kaplan-Meier plots were generated to determine the effect certain DEGs on patient survival (20). The patients were divided into two groups according to the expression level of the gene of interest, and differences in the survival rate were statistically analyzed. The hazard ratio with 95\% confidence intervals and log-rank P-value were calculated and displayed.

Statistical analysis. The numerical data are expressed as the mean \pm standard deviation of at least three experiments. Statistical comparisons between groups of normalized data were performed using Student's t-test or Mann-Whitney U-test according to the test conditions. $\mathrm{P}<0.05$ was considered to indicate a statistically significant difference with a 95\% confidence level. 
A
Normal

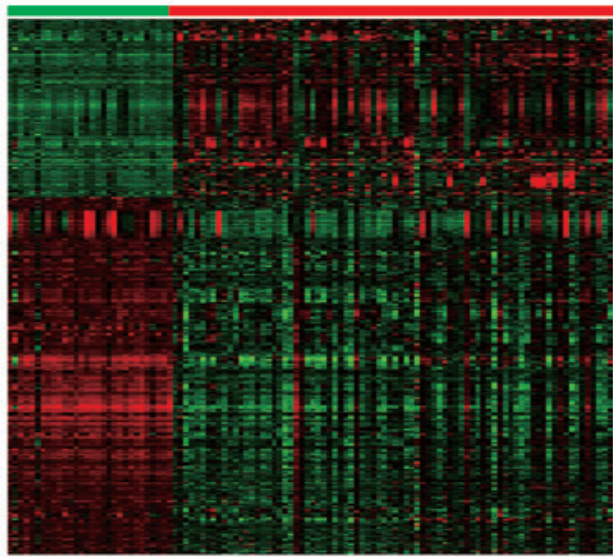

C
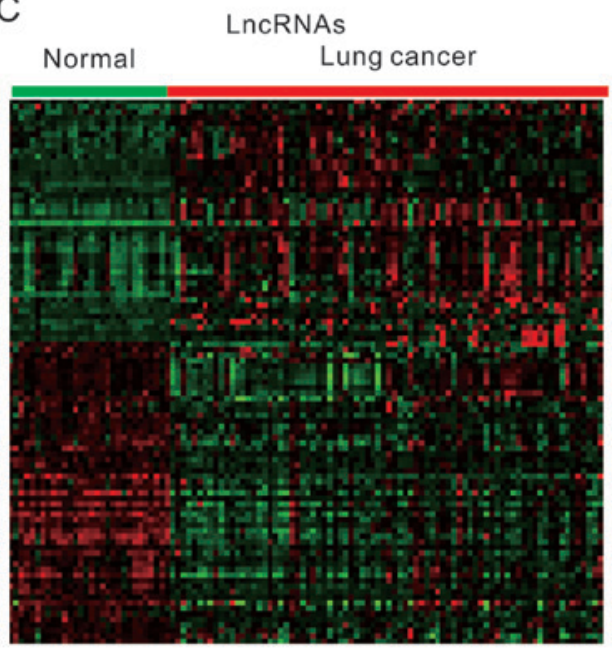

E

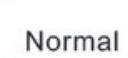

miRNAs

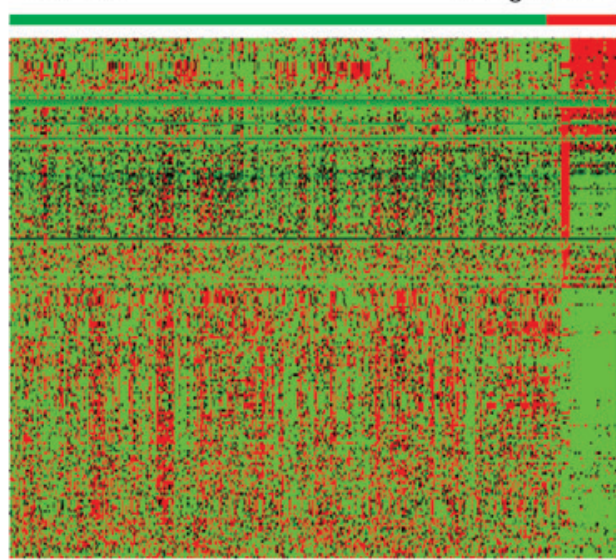

B

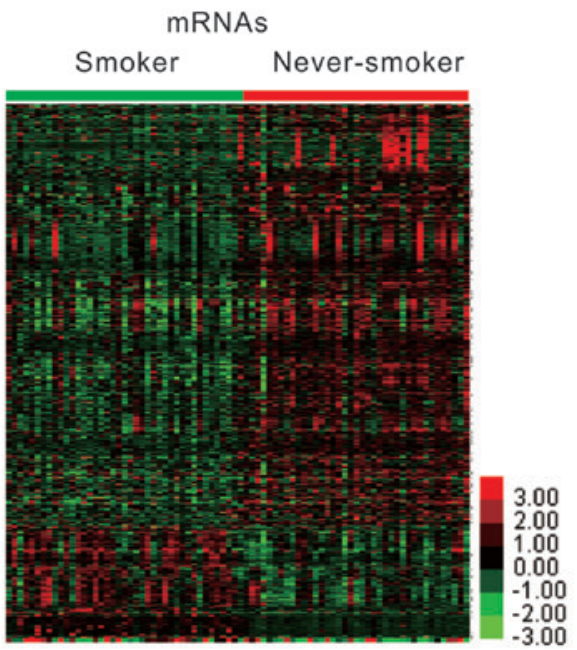

D

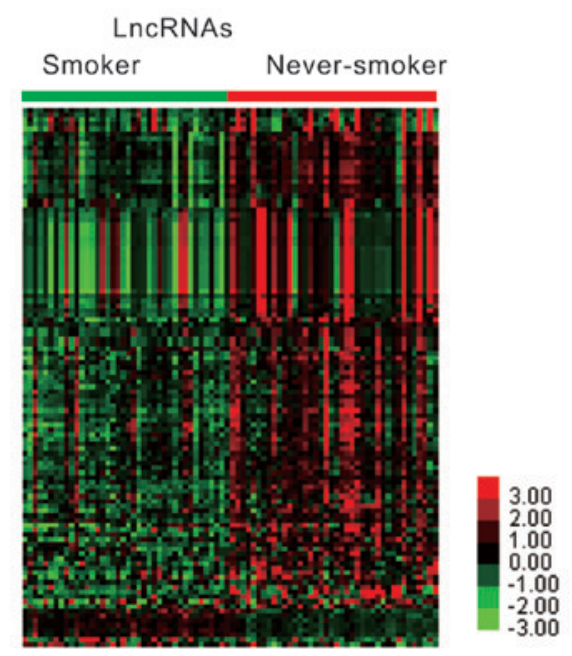

F

miRNAs
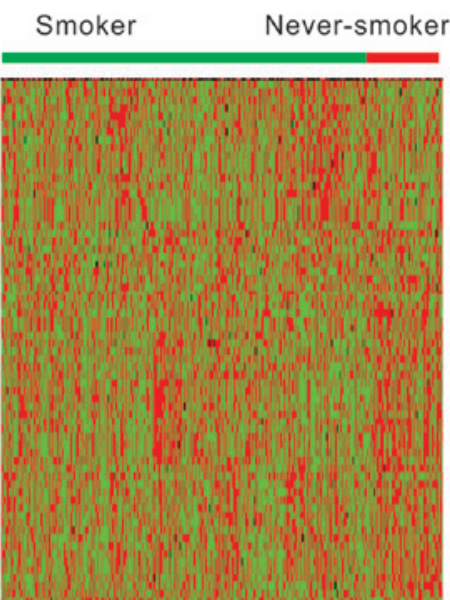

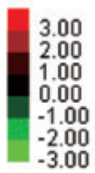

Figure 1. Identification of differently expressed genes, IncRNAs and miRNAs in smoking-associated lung cancer. Hierarchical clustering analysis revealed differentially expressed mRNAs between (A) normal and lung cancer samples and (B) lung cancer samples from smokers and never-smokers from the dataset GSE43458. Hierarchical clustering analysis revealed differential lncRNA expression between (C) normal and lung cancer samples and (D) lung cancer samples from smokers and never-smokers from the dataset GSE43458. Hierarchical clustering analysis reveals differential miRNA expression between (E) normal and lung cancer samples and (F) lung cancer samples from smokers and never-smokers from the dataset TCGA LUAD dataset. IncRNA, long non-coding RNA; miRNA, microRNA.

\section{Results}

Identification of differentially expressed $m R N A s$ in smoking-associated lung cancer. In the present study, the public gene expression dataset GSE43458 from the GEO database was analyzed to identify significantly differentially expressed RNAs between lung cancer and normal lung samples (Fig. 1). Heatmaps generated by hierarchical clustering 


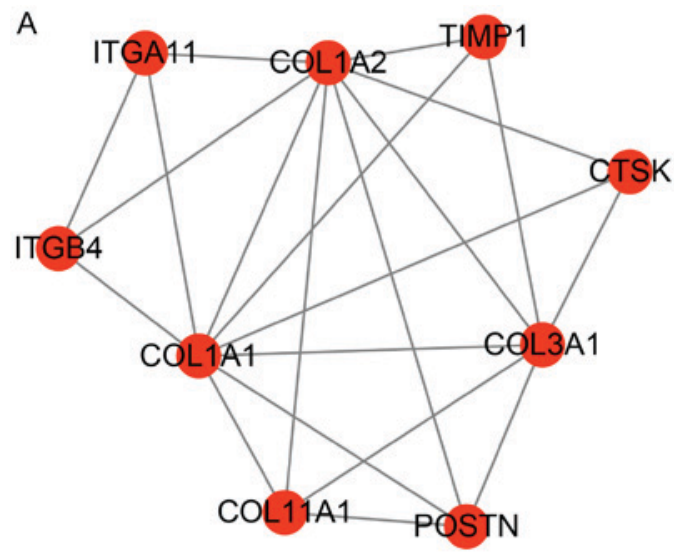

B

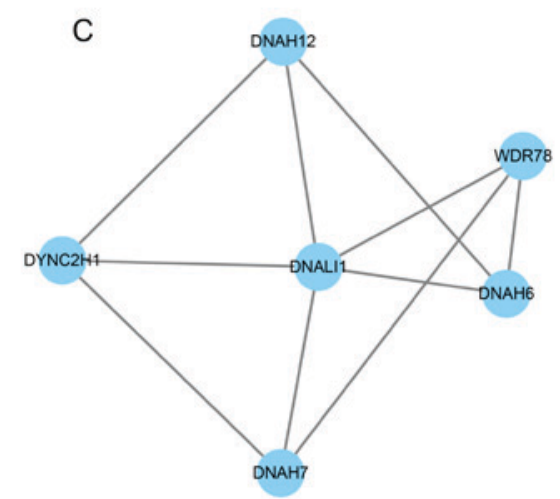

Figure 2. Protein-protein interaction network construction and analysis. (A and B) Two hub-networks were distinct for the upregulated smoking-associated genes and $(\mathrm{C})$ one hub-network was identified for the downregulated smoking-associated genes. Red nodes, upregulated genes; blue nodes, downregulated genes.

analysis of the DEGs in lung cancer are presented in Fig. 1A and B. A total of 729 up- and 1,485 downregulated mRNAs were identified (Fig. 1A). Furthermore, comparison of gene expression patterns between lung cancer samples of smokers and non-smokers identified 610 mRNAs in the GSE43458 dataset (Fig. 1B). smoking-associated

PPI network construction. PPI networks were constructed to predict the interaction association among the 135 up- and 179 downregulated proteins in smoking-associated lung cancer (combined score, $>0.4$ ) by using the STRING database. A module analysis of the network was then performed using the Mcode plugin (degree cut-off, $\geq 2$; nodes with edges, $\geq 2$-core). The PPI networks were constructed by using Cytoscape and presented in Fig. 2. For the upregulated genes in smokingassociated lung cancer, 2 distinct hub networks were identified, while 1 hub network was identified for the downregulated genes in smoking-associated lung cancer. Periostin (POSTN), collagen type III $\alpha 1$ chain (COL3A1), COL1A1, COL1A2, cathepsin K (CTSK), integrin subunit $\beta 4$ (ITGB4), tissue inhibitor of metalloproteinases 1 (TIMP1), ITGA11, COL11A1, MYB proto-oncogene like 2 (MYBL2), karyopherin subunit $\alpha 2$ (KPNA2), aurora kinase A (AURKA), TPX2, microtubule nucleation factor (TPX2) and cell division cycle 20 (CDC20) were identified as key upregulated genes in smokingassociated lung cancer (Fig. 2A and B). Furthermore, a total of 6 genes [dynein axonemal heavy chain 7 (DNAH7), dynein cytoplasmic 2 heavy chain 1 (DYNC2H1), WD repeat domain 78 (WDR78), DNAH6, DNAH12 and dynein axonemal light intermediate chain 1 (DNALI1)] were the key downregulated genes in smoking-associated lung cancer (Fig. 2C).

Functional analysis of deregulated genes in smoking-associated lung cancer. In order to explore the functional roles of the hub genes in smoking-associated lung cancer, the DepMap dataset (https://depmap. org/portal/depmap/) was analyzed. DepMap aims to identify novel diagnostic and therapeutic targets for human cancers by integrating large-scale datasets, including CRISPR-associated protein 9 nuclease screening and small hairpin RNA screening. As presented in Fig. 3, by analyzing CRISPR (Broad Avana) in the DepMap dataset, it was observed that knockout of CTSK, ITGA11, MYBL2, KPNA2, DNAH7, AURKA and TPX2 significantly suppressed $(\mathrm{CERES}<0)$ and knockout of DNAH7 significantly promoted (CERES $>0)$ the proliferation of human cancer cells, including lung cancer.

Influence of deregulated genes in smoking-associated lung cancer on patient survival. To evaluate the prognostic value of 20 hub genes selected by Mcode, Kaplan-Meier-analysis was employed. The median expression level of each target gene among all lung cancer samples was used as the cut-off point to divide all cases into high and low expression groups. The overall survival rates were observed to be lower in AURKA-high compared to AURKA-low expression groups of lung cancer patients, and the same trends were identified for ITGB4, COL1A1, CDC20, MYBL2, POSTN, COL11A1, TPX2, COL3A1 and TIMP1 (P<0.05; Fig. 4A-J). Furthermore, the results indicated that a high mRNA expression of 

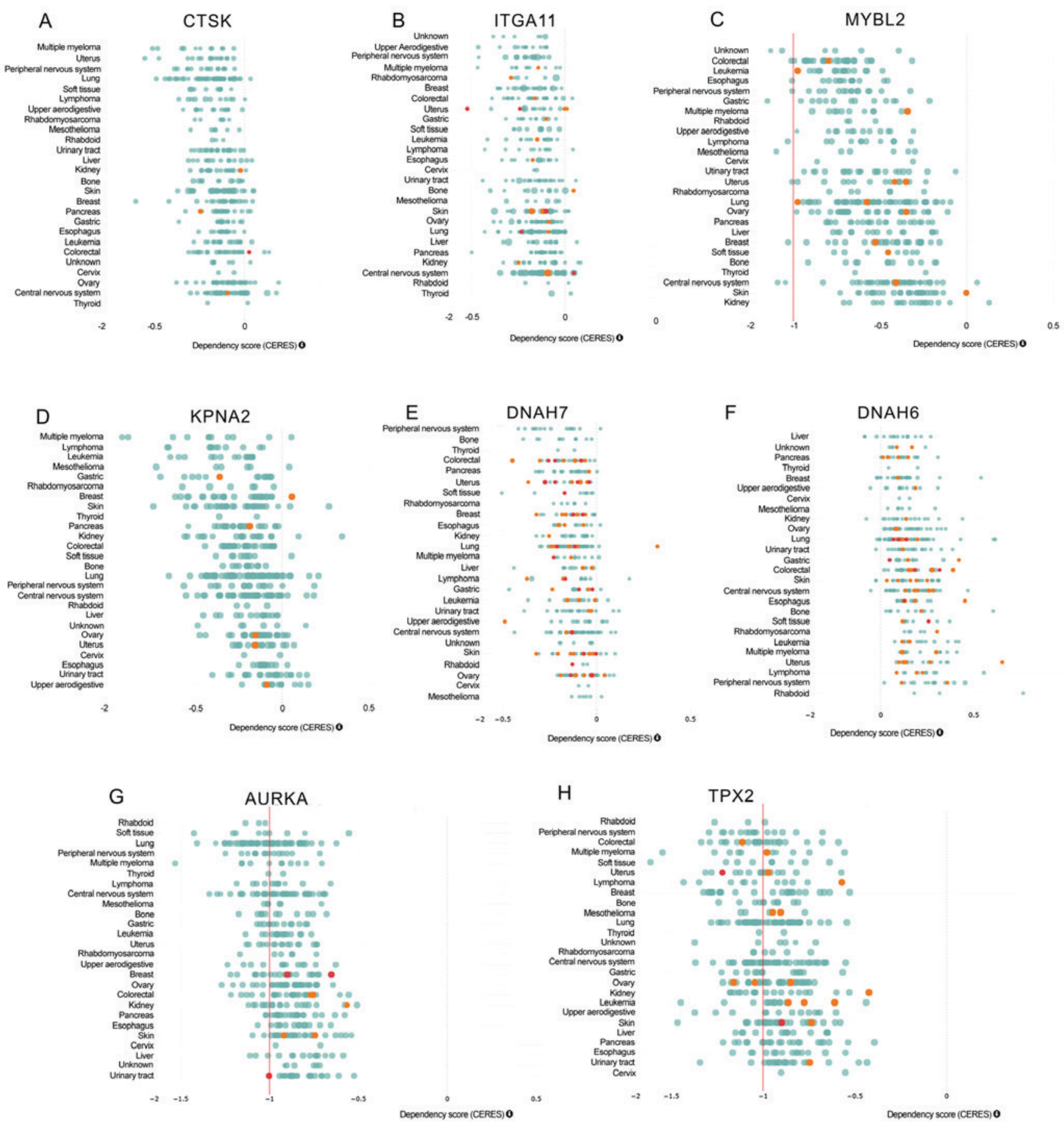

Figure 3. Functional analysis of deregulated genes in smoking-associated lung cancer. The DepMap analysis indicates that knockout of (A) CTSK, (B) ITGA11, (C) MYBL2, (D) KPNA2, (E) DNAH7, (F) AURKA and (G) TPX2 significantly suppressed (CERES $<0$ ) and (H) knockout of DNAH7 significantly promoted (CERES $>0$ ) the proliferation of human cancer cells, including lung cancer. CTSK, cathepsin K; ITGA11, integrin subunit $\alpha$ 11; MYBL2, MYB proto-oncogene like 2; KPNA2, karyopherin subunit $\alpha$ 2; DNAH7, dynein axonemal heavy chain 7; AURKA, aurora kinase A; TPX2, TPX2, microtubule nucleation factor.

DNALI1, DNAH6, CTSK, DYNC2H1, DNAH12 and WDR78 was associated with better overall survival of lung cancer patients (Fig. 4K-P).

Identification of differently expressed lncRNAs in smoking-associated lung cancer. Various lncRNAs have been revealed to be associated with the progression of numerous different types of human cancer, including lung cancer. However, few studies have focused on smoking-associated IncRNAs in lung cancer. In the present study, an IncRNA classification pipeline was used to identify differently expressed IncRNAs in smoking-associated lung cancer. Heatmaps generated by hierarchical clustering analysis of the differentially expressed lncRNAs in lung cancer are presented in Fig. $1 \mathrm{C}$ and D.

Co-expression network analysis of lncRNAs in smoking-associated lung cancer. To predict the potential functional roles of these lncRNAs, the Pearson correlation coefficient of IncRNA-mRNA pairs was first calculated. The co-expressed mRNA-lncRNA pairs with an absolute value of the Pearson correlation coefficient of $\geq 0.75$ 

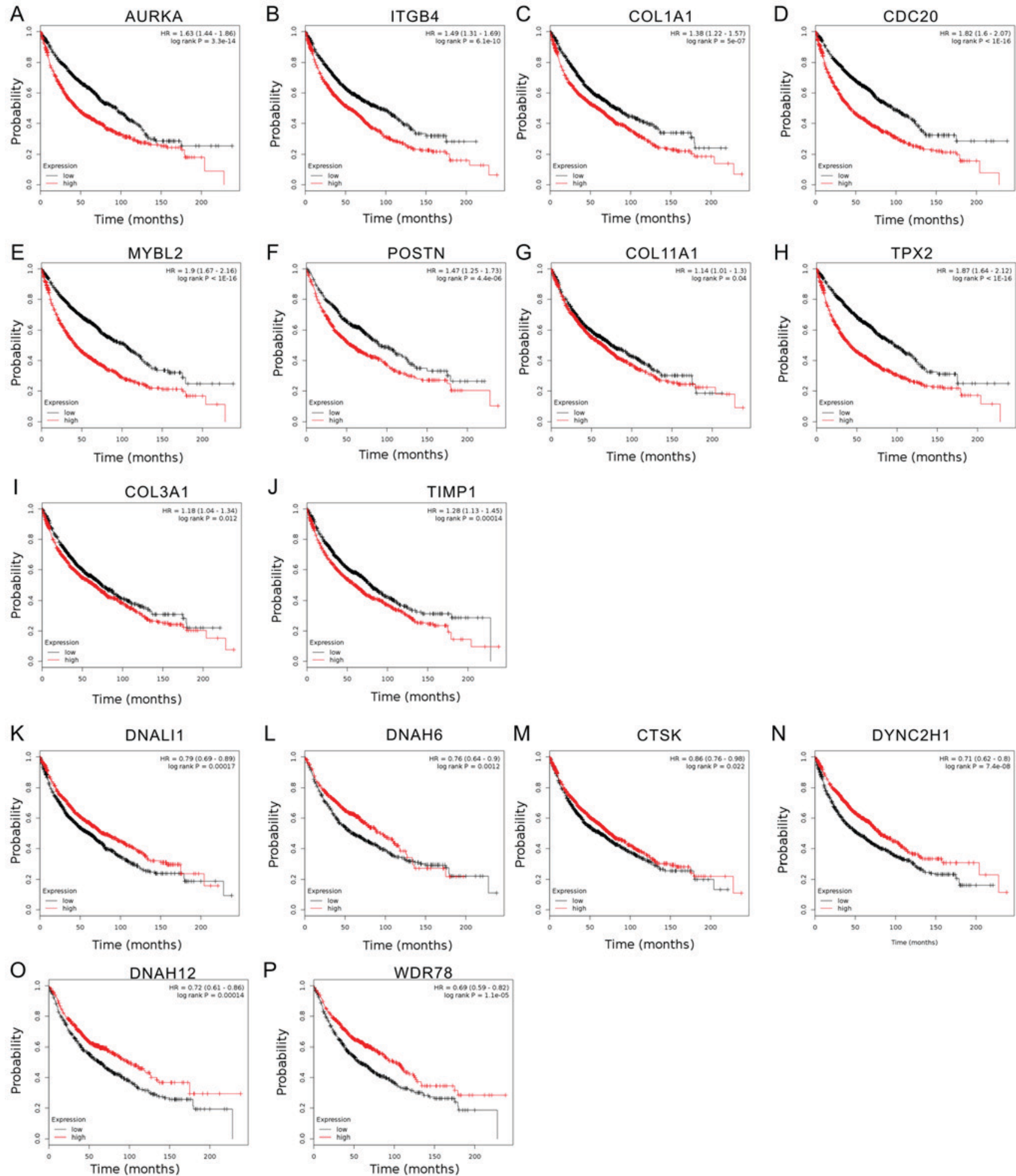

Figure 4. Analysis of the influence of deregulated genes in smoking-associated lung cancer on survival of lung cancer patients. Kaplan-Meier analysis of the effect of 16 hub genes, including (A) AURKA, (B) ITGB4, (C) COL1A1, (D) CDC20, (E) MYBL2, (F) POSTN, (G) COL11A1, (H) TPX2, (I) COL3A1, (J) TIMP1, (K) DNALI1, (L) DNAH6, (M) CTSK, (N) DYNC2H1, (O) DNAH12 and (P) WDR78, on the survival of lung cancer patients. AURKA, aurora kinase A; ITGB4, integrin subunit $\beta$ 4; COL3A1, collagen type III $\alpha 1$ chain; CDC20, cell division cycle 20; MYBL2, MYB proto-oncogene like 2; POSTN, periostin; TPX2, TPX2, microtubule nucleation factor; TIMP1, tissue inhibitor of metalloproteinases 1; DNAH6, dynein axonemal heavy chain 6; DNALI1, dynein axonemal light intermediate 1; CTSK, cathepsin K; DYNC2H1, dynein cytoplasmic 2 heavy chain 1; WDR78, WD repeat domain 78.

were selected to construct co-expression networks with Cytoscape. The network presented in Fig. 5 includes 24 lncRNAs and 580 mRNAs. Certain lncRNAs, including relaxin family peptide receptor 1 (RXFP1), receptor activity modifying protein 2 -antisense RNA 1
(RAMP2-AS1), LINC00312 and LINC00472, were co-expressed with $>100$ mRNAs with an absolute value of the Pearson correlation coefficient of $\geq 0.75$. These lncRNAs were identified as key lncRNAs in smoking-associated lung cancer. 


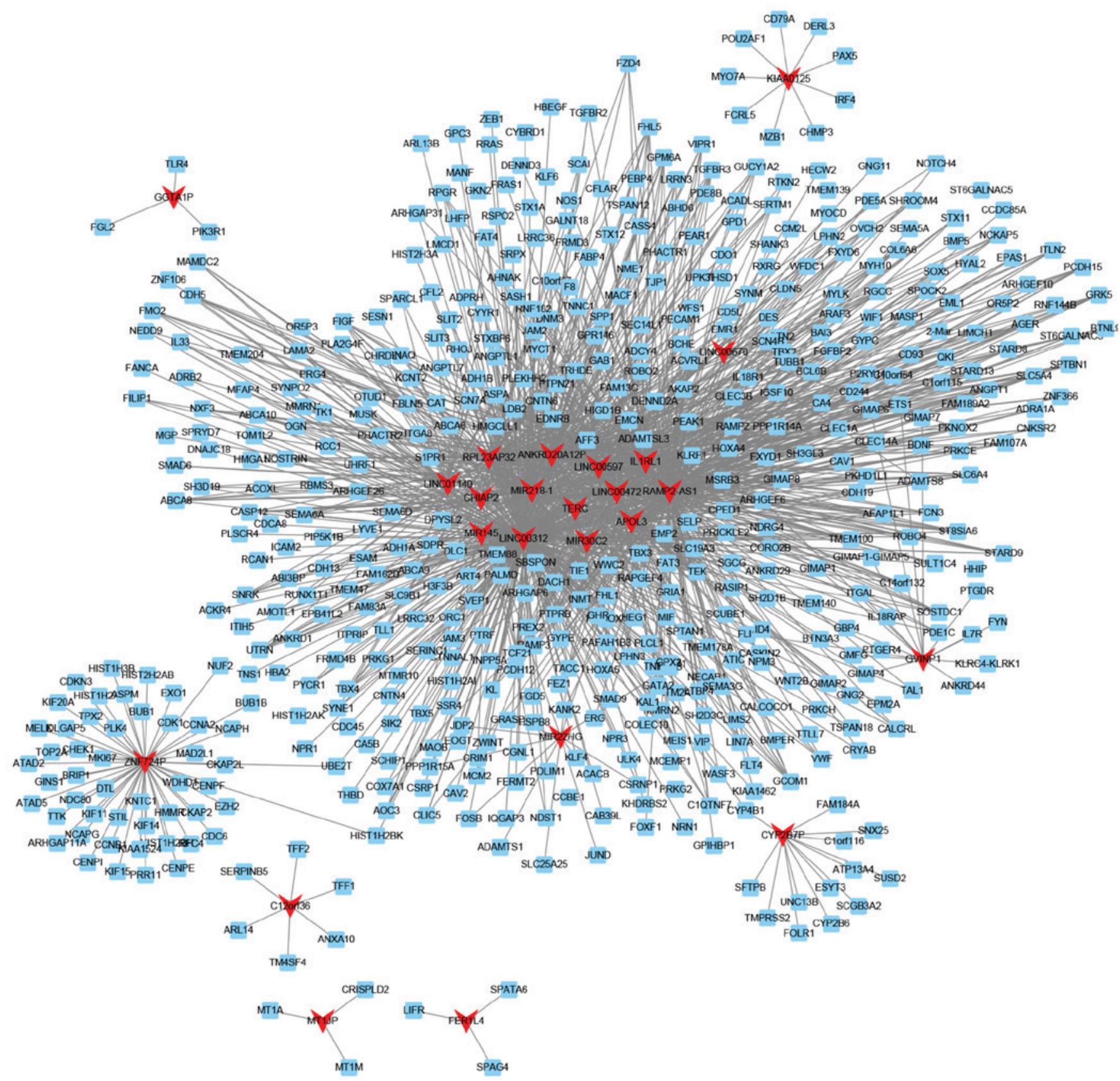

Figure 5. Co-expression network analysis of lncRNA-mRNA pairs in smoking-associated lung cancer. Red nodes represent lncRNA and blue nodes represent mRNA. lncRNA, long non-coding RNA.

$G O$ and $K E G G$ analysis of deregulated lncRNAs in smoking-associated lung cancer. Furthermore, GO and KEGG enrichment analyses were performed for the differentially expressed lncRNAs (Fig. 5). GO analysis revealed that lncRNAs that were deregulated in smoking-associated lung cancer were mainly involved in regulating vasculogenesis, cell adhesion, sister chromatid cohesion, angiogenesis, receptor internalization, cell division, leukocyte migration, cell proliferation, mitotic spindle organization and mitotic cell cycle checkpoint (Fig. 6A). Furthermore, it was identified that the deregulated lncRNAs were enriched in GO terms in the category molecular function associated with protein binding, protein heterodimerization activity, growth factor binding, actin filament binding and transforming growth factor $\beta$-activated receptor activity (Fig. 6B).
KEGG pathway analysis revealed that the deregulated lncRNAs were primarily enriched in pathways associated with vascular smooth muscle contraction, alcoholism, cell cycle, cyclic guanosine monophosphate cGMP)/protein kinase cGMP-dependent 1 signaling pathway, renin secretion, malaria, tight junction, regulation of lipolysis in adipocytes, circadian entrainment and long-term depression (Fig. 6C).

Key lncRNAs downregulated in smoking-associated lung cancer. In the present study, RXFP1, RAMP2-AS1, LINC00312 and LINC00472 were identified as key lncRNAs in smoking-associated lung cancer. However, their prognostic value and functional roles in lung cancer have remained elusive. Therefore, their specific expression patterns were analyzed in other public datasets from TCGA. 
A Smoking-associated IncRNAs related biological processes

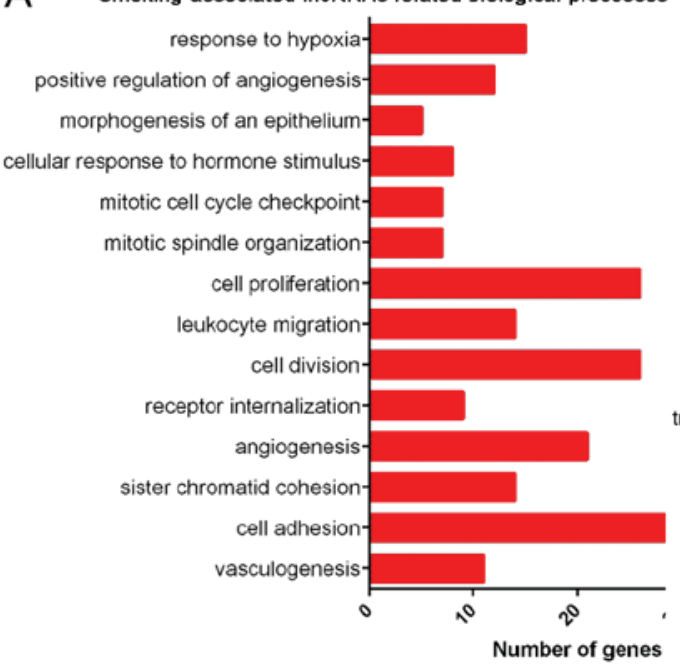

B

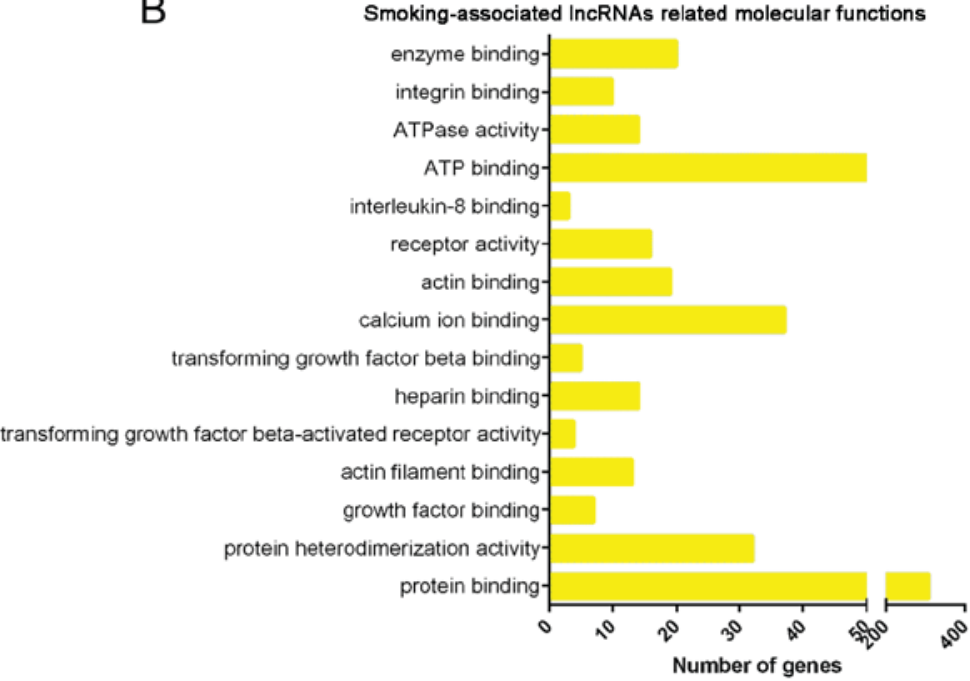

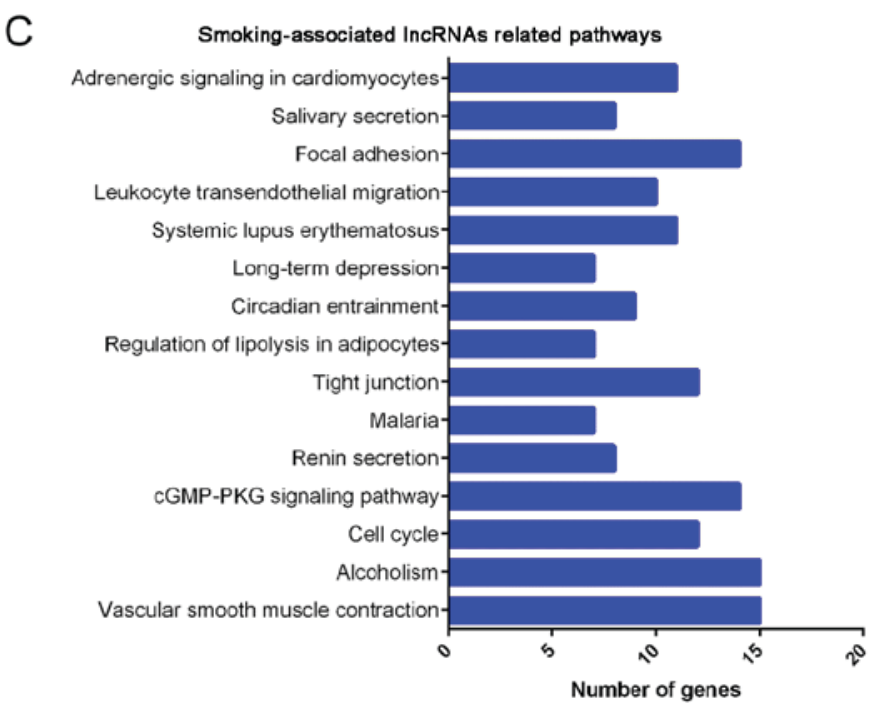

Figure 6. GO and KEGG analysis of deregulated lncRNAs in smoking- related lung cancer. Deregulated lncRNAs enriched in (A and B) GO terms in the categories (A) biological process and (B) molecular function, and in (C) KEGG pathways. GO, gene ontology; KEGG, Kyoto Encyclopedia of Genes and Genomes; lncRNA, long non-coding RNA; cGMP, cyclic guanosine monophosphate; PKG, protein kinase.

The results indicated that RXFP1, RAMP2-AS1, LINC00312 and LINC00472 may act as tumor suppressors and were significantly downregulated in LUAD (Fig. 7). Furthermore, Kaplan-Meier-analysis was used to reveal their potential prognostic value. As presented in Fig. 7, higher expression levels of RXFP1, RAMP2-AS1, LINC00312 and LINC00472 were significantly associated with a longer overall survival time (Fig. 8).

Identification of differently expressed miRNAs in smoking-associated lung cancer. Emerging studies have demonstrated that miRNAs have crucial roles in lung cancer. In the present study, the TCGA LUAD dataset was analyzed to identify differently expressed miRNAs in smoking-associated lung cancer. A total of 53 miRNAs were identified to be dysregulated in lung cancer samples of smokers. Furthermore, 107 miRNAs were identified to be upregulated and 101 miRNAs were indicated to be downregulated in lung cancer compared with normal samples (Fig. 1E-F). Finally, Homo sapiens (hsa)-miR-3934, hsa-miR-101-1, hsa-miR-30e and hsa-miR-190 were identified as differently expressed miRNAs in smoking-associated lung cancer (Fig. 9).

\section{Discussion}

Lung cancer is the leading cause of cancer-associated mortalities worldwide. Smoking is one of the most significant etiological contributors to lung cancer development (21). However, the molecular mechanisms underlying smoking-induced initiation and progression of lung cancer have remained largely elusive. In present study, the public dataset GSE43458 was analyzed, and 135 up- and 179 downregulated mRNAs in smokingassociated lung cancer were identified. Furthermore, PPI networks were constructed to identify hub genes. A total of 6 downregulated genes (DNAH7, DYNC2H1, WDR78, DNAH6, DNAH12 and DNALI1) and 14 upregulated genes (POSTN, COL3A1, COL1A1, COL1A2, CTSK, ITGB4, TIMP1, ITGA11, COL11A1, MYBL2, KPNA2, AURKA, TPX2 and CDC20) were identified as hub genes in 

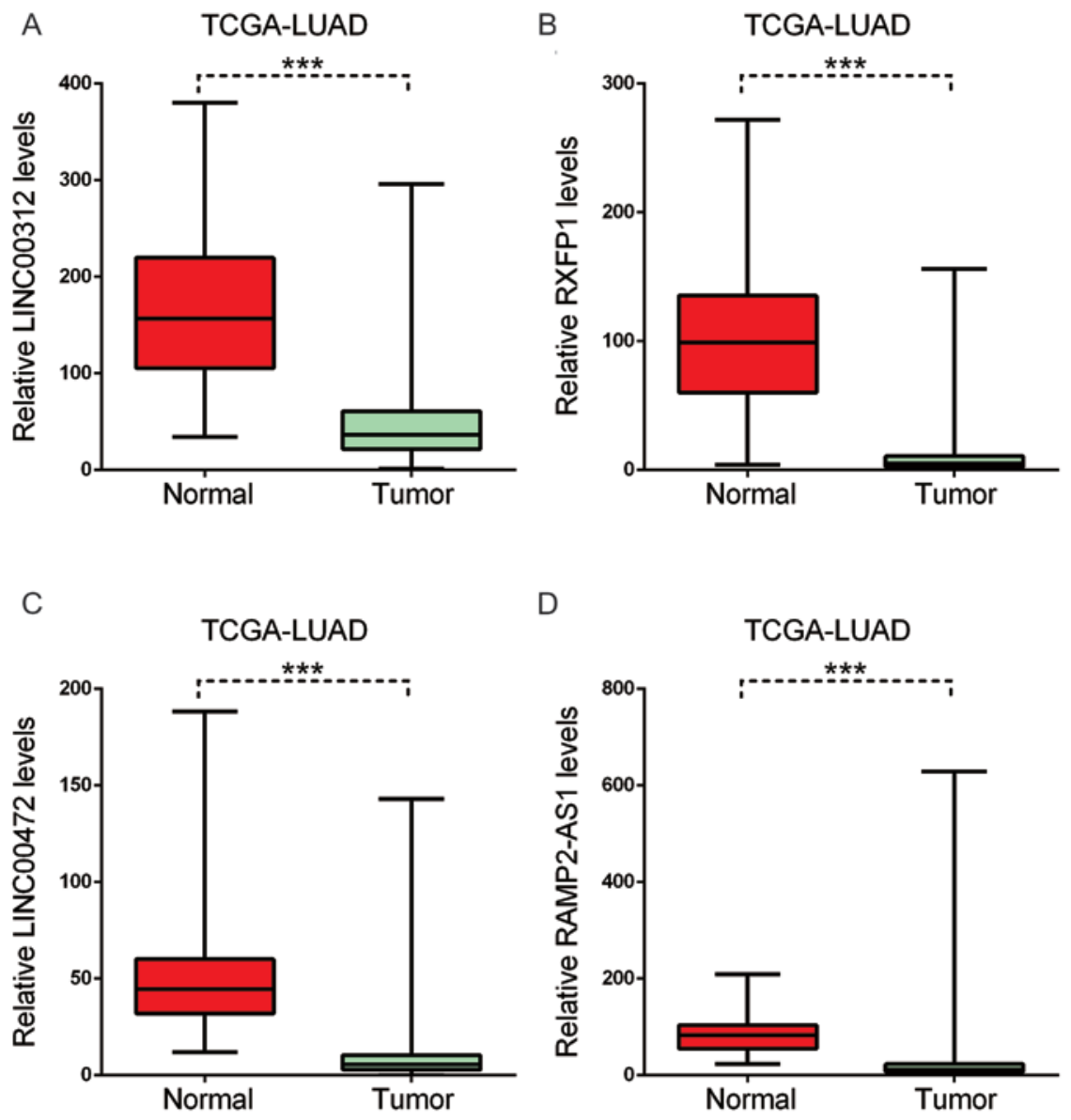

Figure 7. Key long non-coding RNAs in smoking-associated lung cancer were downregulated. In LUAD, (A) LINC00312, (B) RXFP1, (C) LINC00472 and (D) RAMP2-AS1 were downregulated. Boxplots indicate the minimum, maximum, median and upper and lower quartiles of gene expression levels in each group. The red boxplot indicates gene expression in normal groups, the blue boxplot indicates gene expression in tumor groups. ${ }^{* * *} \mathrm{P}<0.001$. LUAD, lung adenocarcinoma; LUSC, lung squamous cell carcinoma; TCGA, The Cancer Genome Atlas; RXFP1, relaxin family peptide receptor 1; RAMP2-AS1, receptor activity modifying protein 2-antisense RNA 1; LINC00312, long intergenic non-protein coding RNA 312.

smoking-associated lung cancer. Of note, AURKA has been previously reported to be upregulated in the tumor tissues of smoking patients (22). Furthermore, the DepMap dataset was analyzed to evaluate the potential functions of these hub genes, revealing that CTSK, ITGA11, MYBL2, KPNA2, DNAH7, AURKA, TPX2 and DNAH7 are involved in regulating the proliferation of human cancer cells, including lung cancer. Most of these hub genes were identified to be associated with smoking-associated lung cancer for the first time in the present study, to the best of our knowledge.

miRNAs and lncRNAs have been indicated to serve as important regulators in various diseases, including lung cancer, by affecting various biological processes, including cell cycle, apoptosis and invasion. For instance, lncRNA small nucleolar RNA host gene 20 was reported to promote NSCLC cell proliferation and migration by epigenetically silencing P21 expression (23). IncRNA MetaLnc9 was also reported to facilitate lung cancer metastasis via the phosphoglycerate kinase 1-activated AKT/mammalian target of rapamycin pathway (24). Furthermore, several lncRNAs, including MALAT1, CCAT1 and LINC00094, were identified to be associated with smoking-induced lung cancer (12). In present study, differently expressed genes were screened in lung cancer tissues of smokers vs. non-smokers. A total of 11 up- and 13 downregulated lncRNAs in smoking-associated lung cancer were identified in the present study. TCGA LUAD dataset was screened to identify hsa-miR-3934, hsa-miR-101-1, hsa-miR-30e and hsa-miR-190 as differentially expressed miRNAs in smoking-associated lung cancer. Furthermore, a co-expression network analysis revealed that certain key lncRNAs, including RXFP1, RAMP2-AS1, LINC00312 and LINC00472. A GO and KEGG enrichment analysis indicated that these IncRNAs were associated with vasculogenesis, cell adhesion, sister chromatid cohesion, angiogenesis, receptor internalization, cell division, cell proliferation, cell cycle and cGMP/PKG signaling pathway.

In previous studies, LINC00312 and LINC00472 have been indicated to be associated with lung cancer progression. For instance, Zhu et al (25) reported that LINC00312 was downregulated in NSCLC tissues and correlated with a poor clinical outcome. Functional experiments indicated that LINC00312 may inhibit cell proliferation and promote apoptosis in vitro and in vivo. Furthermore, Tian et al (26) observed that LINC00312 is downregulated in lung cancer. The functional roles of LINC00472 in lung cancer have been revealed by bioinformatics analyses. For instance, Sui et al (27) and Zhu et al (28) reported that LINC00472 was downregulated in lung cancer by constructing an IncRNA-mediated competitive endogenous RNA network. To the best of our knowledge, the present study was the first to identify that LINC00312 
A

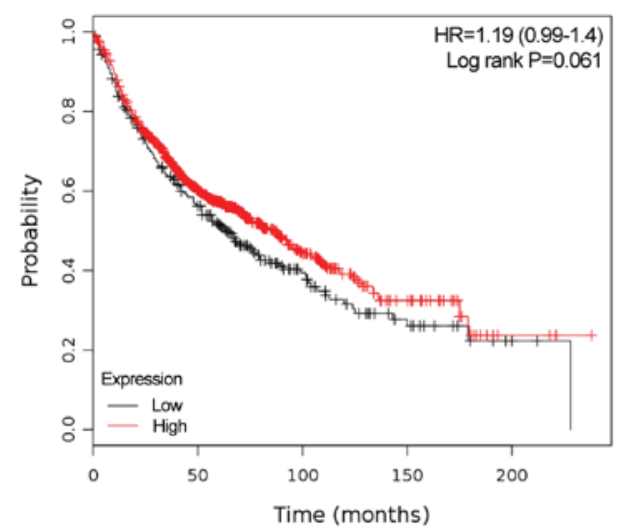

C

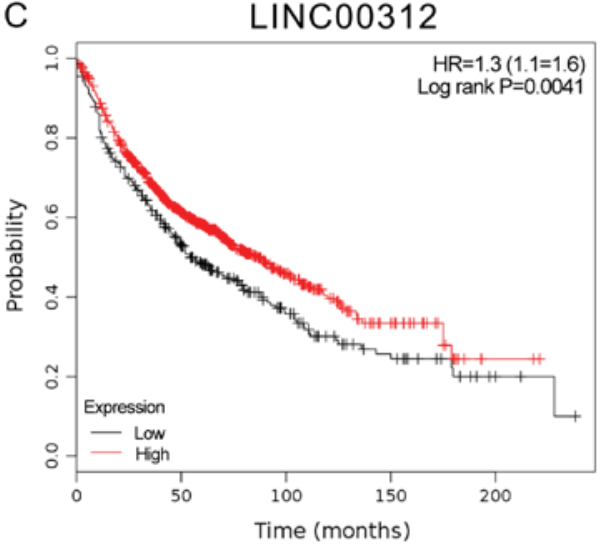

B

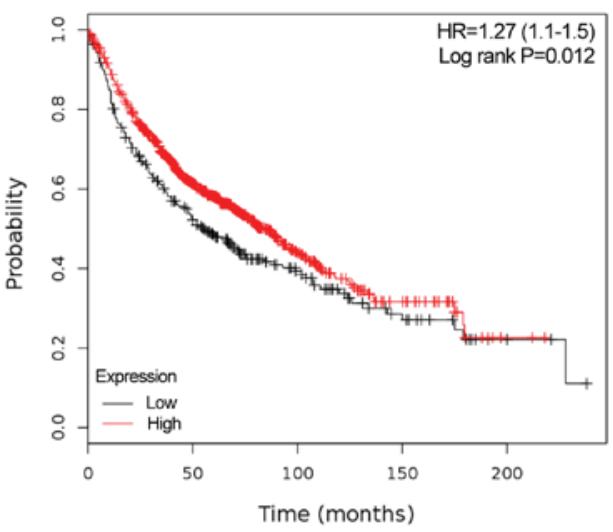

D

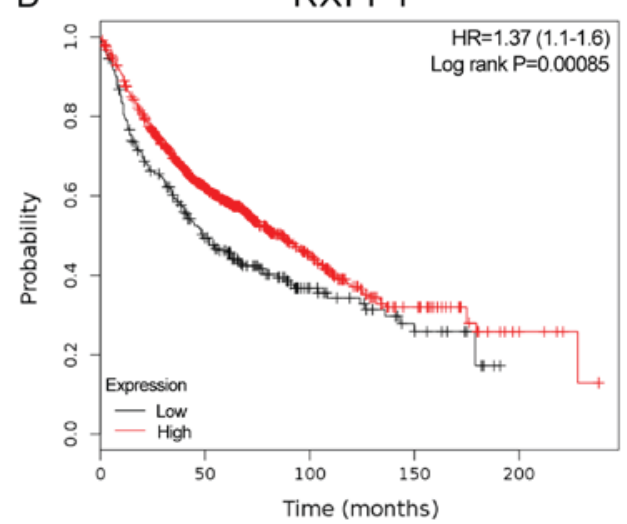

Figure 8. Survival analysis of deregulated lncRNAs in smoking-associated lung cancer. (A) The Kaplan-Meier curve analysis indicates that higher expression levels of LINC00472 was weakly associated with a longer overall survival time. (B and C) (B) RAMP2-AS1, (C) LINC00312 and (D) RXFP1 were significantly associated with a longer overall survival time. The median expression of LINC00472, RAMP2-AS1, LINC00312 and RXFP1 were selected as a cutoff to stratify patients into high and low expression groups. The HR values with 95\% confidence intervals are provided for the respective lncRNAs. RXFP1, relaxin family peptide receptor 1; RAMP2-AS1, receptor activity modifying protein 2-antisense RNA 1; LINC00312, long intergenic non-protein coding RNA 312; HR, hazard ratio; lncRNA, long non-coding RNA.

and LINC00472 are associated with smoking-induced lung cancer.

In the present study, the prognostic value of the hub genes and lncRNAs in lung cancer was determined. Kaplan-Meier analysis revealed that high mRNA expression of DNAH7, DYNC2H1, WDR78, DNAH6, DNAH12 and DNALI1 was associated with a longer overall survival time in lung cancer patients. However, the overall survival time of lung cancer patients with high POSTN expression were shorter compared with those with low POSTN expression, and the same trends were identified for COL3A1, COL1A1, COL1A2, CTSK, ITGB4, TIMP1, ITGA11, COL11A1, MYBL2, KPNA2, AURKA, TPX2 and CDC20. Conversely, higher expression levels of lncRNA RXFP1, RAMP2-AS1, LINC00312 and LINC00472 were significantly associated with a longer overall survival time. These results suggested that these RNAs may provide novel tools for the diagnosis and prognostication, as well as drug targets for smoking-associated lung cancer.

Of note, the present study has several limitations. First, the expression pattern of key regulatory RNAs in smoking-associated lung cancer should be further validated. The correlation between the expression of regulatory RNAs in smoking-associated lung cancer and clinicopathological features of the patients, including age, sex, Grade, T stage,
$\mathrm{N}$ stage, smoking status and survival status, should be further evaluated. Furthermore, it was demonstrated that smoking-associated genes predicted the outcome of NSCLC patients. However, it may be appropriate to assess whether the dysregulation of smoking-associated genes are associated with smoking and non-smoking patients with NSCLC. This may be problematic as the number of non-smoking patients with NSCLC is limited. In addition, previous studies have demonstrated that the competing endogenous RNA network (ceRNA) serves crucial roles in cancer progression. In the current study, smoking associated lncRNAs, miRNAs and mRNAs were identified. Therefore, the construction of smoking associated ceRNA networks in NSCLC may provide useful information to understand the potential mechanisms that underly cancer progression. In addition, the functional roles of these regulatory genes should be further validated by performing loss/gain of function assays.

In conclusion, the present bioinformatics study identified 314 mRNAs, 24 lncRNAs and 4 miRNAs that are deregulated in smoking-associated NSCLC. PPI network analysis identified 20 hub genes in smoking-associated lung cancer, including DNAH7, DYNC2H1, WDR78, COL3A1, COL1A1 and COL1A2. Co-expression network analysis indicated that RXFP1, RAMP2-AS1, LINC00312 and LINC00472 are key 

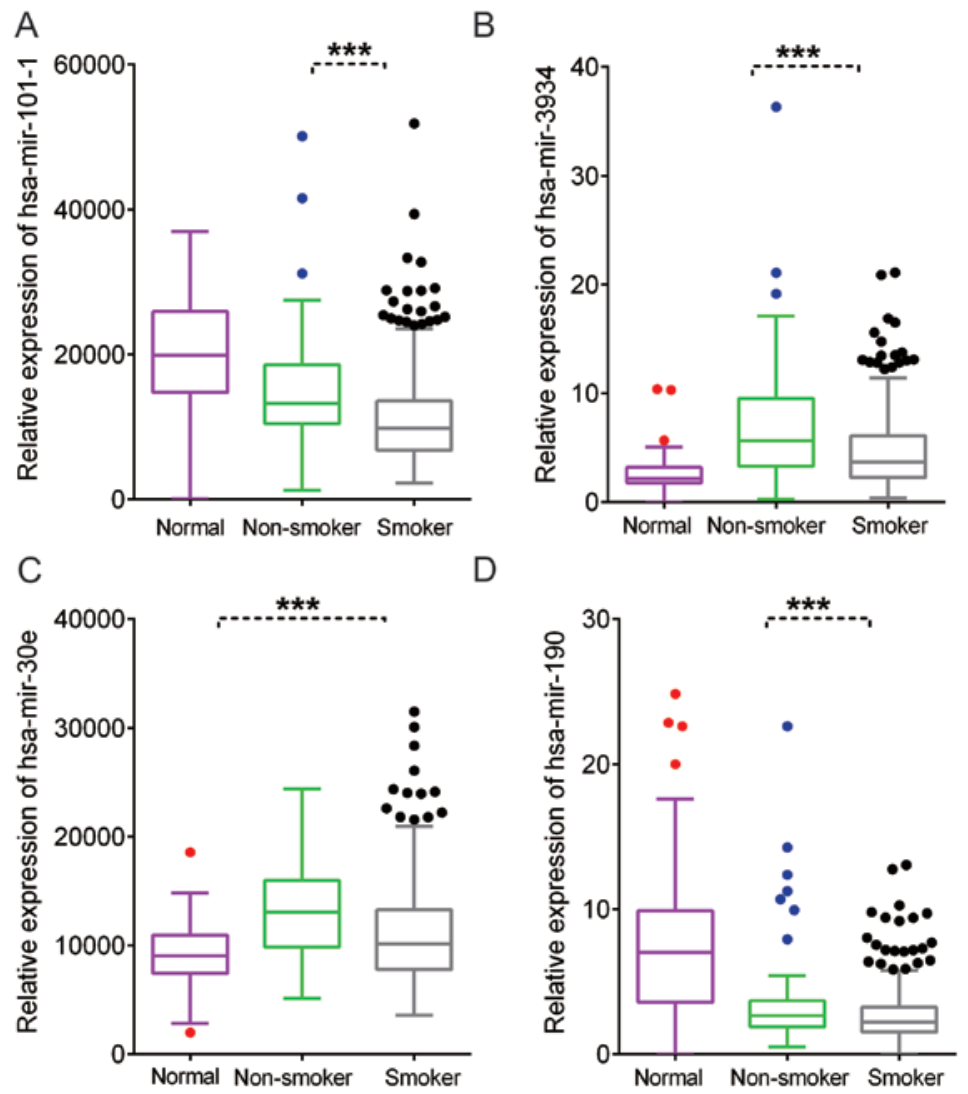

Figure 9. Relative expression of key miRNAs in lung cancer. (A-D) Relative expression of (A) hsa-miR-101-1, (B) hsa-miR-3934, (C) hsa-miR-30e and (D) hsa-miR-190 in normal samples, lung cancer samples from non-smokers and smokers. Boxplots indicate the minimum, maximum, median and upper and lower quartiles of gene expression levels in each group. The red boxplot indicates gene expression in normal groups and the blue boxplot indicates gene expression in tumor groups ${ }^{* * *} \mathrm{P}<0.001$. miR, microRNA; hsa, Homo sapiens.

lncRNAs. Furthermore, GO and KEGG analysis indicated that these smoking-associated lncRNAs are enriched in a variety of functions and pathways, including cell proliferation and the cGMP/PKG signaling pathway. Of note, these hub genes and lncRNAs were associated with the prognosis of lung cancer patients. Although further validation of the present results is required, the present study provides useful information to further explore potential candidate biomarkers for the diagnosis, prognostication and utilization as drug targets for smoking-associated lung cancer.

\section{Acknowledgements}

Not applicable.

\section{Funding}

No funding received.

\section{Availability of data and materials}

All data generated or analyzed during this study are included in this published article.

\section{Authors' contributions}

$\mathrm{XD}$ and $\mathrm{YC}$ conceived and designed the present study. YC, YP and YJ collected and assembled the data. YC, LS and XD analyzed and interpreted the data. All authors contributed to writing the manuscript and approved the final version.

\section{Ethics approval and consent to participate}

Not applicable.

\section{Patient consent for publication}

Not applicable.

\section{Competing interests}

The authors declare that they have no competing interests.

\section{References}

1. Bhattacharjee A, Richards WG, Staunton J, Li C, Monti S, Vasa P, Ladd C, Beheshti J, Bueno R, Gillette M, et al: Classification of human lung carcinomas by mRNA expression profiling reveals distinct adenocarcinoma subclasses. Proc Natl Acad Sci USA 98: 13790-13795, 2001.

2. Molina JR, Yang PG, Cassivi SD, Schild SE and Adjei AA: Non-small cell lung cancer: Epidemiology, risk factors, treatment, and survivorship. Mayo Clin Pro 83: 584-594, 2008.

3. Roggli VL, Vollmer RT, Greenberg SD, Mcgavran MH, Spjut HJ and Yesner R: Lung-Cancer heterogeneity: A blinded and randomized study of 100 consecutive cases. Hum Pathol 16: 569-579, 1985.

4. Bryant A and Cerfolio RJ: Differences in epidemiology, histology, and survival between cigarette smokers and never-smokers who develop non-small cell lung cancer. Chest 132: 185-192, 2007. 
5. Lv XJ, Du YW, Hao YQ, Su ZZ, Zhang L, Zhao LJ and Zhang J: RNA-binding motif protein 5 inhibits the proliferation of cigarette smoke-transformed BEAS-2B cells through cell cycle arrest and apoptosis. Oncol Rep 35: 2315-2327, 2016.

6. Hamdy SI, Hiratsuka M, Narahara K, Endo N, El-Enany M, Moursi N, Ahmed MSE and Mizugaki M: Genotyping of four genetic polymorphisms in the CYP1A2 gene in the Egyptian population. Br J Clin Pharmacol 55: 321-324, 2003.

7. Mattick JS and Makunin IV: Non-coding RNA. Hum Mol Genet 15: R17-R29, 2006.

8. Wan XC, Huang WH, Yang S, Zhang YL, Pu HL, Fu FQ, Huang Y, $\mathrm{Wu} \mathrm{H}, \mathrm{Li} \mathrm{T}$ and $\mathrm{Li} \mathrm{Y}$ : Identification of androgen-responsive lncRNAs as diagnostic and prognostic markers for prostate cancer. Oncotarget 7: 60503-60518, 2016.

9. Kornienko AE, Guenzl PM, Barlow DP and Pauler FM. Gene regulation by the act of long non-coding RNA transcription. BMC Biol 11: 2013.

10. Sun Y, Hu B, Wang Q, Ye M, Qiu Q, Zhou Y, Zeng F, Zhang X, Guo Y and Guo L: Long non-coding RNA HOTTIP promotes BCL-2 expression and induces chemoresistance in small cell lung cancer by sponging miR-216a. Cell Death Dis 9: 85, 2018.

11. Lin A, Hu Q, Li C, Xing Z, Ma G, Wang C, Li J, Ye Y, Yao J, Liang K, et al: The LINK-A lncRNA interacts with PtdIns $(3,4,5)$ $\mathrm{P} 3$ to hy peractivate AKT and confer resistance to AKT inhibitors. Nat Cell Biol 19: 238-251, 2017.

12. Li SC, Sun X, Miao SC, Liu J and Jiao WJ: Differential protein-coding gene and long noncoding RNA expression in smoking-related lung squamous cell carcinoma. Thorac Cancer 8: 672-681, 2017.

13. Kabbout M, Garcia MM, Fujimoto J, Liu DD, Woods D, Chow CW, Mendoza G, Momin AA, James BP, Solis L, et al: ETS2 mediated tumor suppressive function and MET oncogene inhibition in human non-small cell lung cancer. Clin Cancer Res 19: 3383-3395, 2013.

14. Page RD: TreeView. Glasgow University, Glasgow, UK, 2001.

15. Yang J, Lin J, Liu T, Chen T, Pan S, Huang W and Li S: Analysis of lncRNA expression profiles in non-small cell lung cancers (NSCLC) and their clinical subtypes. Lung Cancer 85: 110-115, 2014.

16. Szklarczyk D, Franceschini A, Kuhn M, Simonovic M, Roth A, Minguez P, Doerks T, Stark M, Muller J, Bork P, et al: The STRING database in 2011: functional interaction networks of proteins, globally integrated and scored. Nucleic Acids Res 39: D561-D568, 2011

17. Shannon P, Markiel A, Ozier O, Baliga NS, Wang JT, Ramage D, Amin N, Schwikowski B and Ideker T. Cytoscape: A software environment for integrated models of biomolecular interaction networks. Genome Res 13: 2498-2504, 2003.
18. Bader GD and Hogue CW: An automated method for finding molecular complexes in large protein interaction networks. BMC Bioinformatics 4: 2, 2003.

19. Assenov Y, Ramirez F, Schelhorn SE, Lengauer $T$ and Albrecht M: Computing topological parameters of biological networks. Bioinformatics 24: 282-284, 2008.

20. Győrffy B, Surowiak P, Budczies J and Lánczky A: Online survival analysis software to assess the prognostic value of biomarkers using transcriptomic data in non-small-cell lung cancer. PLoS One 8: e82241, 2013.

21. Hecht SS: Cigarette smoking and lung cancer: Chemical mechanisms and approaches to prevention. Lancet Oncol 3: 461-469, 2002.

22. Zhang J, Li BG, Yang Q, Zhang PY and Wang HT: Prognostic value of Aurora kinase A (AURKA) expression among solid tumor patients: A systematic review and meta-analysis. Jpn J Clin Oncol 45: 629-636, 2015.

23. Chen ZY, Chen X, Chen P, Yu SX, Nie FQ, Lu BB, Zhang T, Zhou Y, Chen QN, Wei CC, et al: Long non-coding RNA SNHG20 promotes non-small cell lung cancer cell proliferation and migration by epigenetically silencing of P21 expression. Cell Death Dis 8: e3092, 2017.

24. Yu T, Zhao YJ, Hu ZX, Li J, Chu DD, Zhang JW, Li Z, Chen B, Zhang X, Pan HY, et al: MetaLnc9 facilitates lung cancer metastasis via a PGK1-activated AKT/mTOR pathway. Cancer Res 77: 5782-5794, 2017

25. Zhu Q, Lv T, Wu Y, Shi X, Liu H and Song Y: Long non-coding RNA 00312 regulated by HOXA5 inhibits tumour proliferation and promotes apoptosis in Non-small cell lung cancer. J Cell Mol Med 21: 2184-2198, 2017.

26. Tian Z, Wen S, Zhang Y, Shi X, Zhu Y, Xu Y, Lv H and Wang G: Identification of dysregulated long non-coding RNAs/microRNAs/mRNAs in TNM I stage lung adenocarcinoma. Oncotarget 8: 51703-51718, 2017.

27. Sui J, Li YH, Zhang YQ, Li CY, Shen X, Yao WZ, Peng H, Hong WW, Yin L, Pu YP and Liang GY: Integrated analysis of long non-coding RNA-associated ceRNA network reveals potential lncRNA biomarkers in human lung adenocarcinoma. Int J Oncol 49: 2023-2036, 2016.

28. Zhu TG, Xiao X, Wei Q, Yue M and Zhang LX: Revealing potential long non-coding RNA biomarkers in lung adenocarcinoma using long non-coding RNA-mediated competitive endogenous RNA network. Braz J Med Biol Res 50: e6297, 2017.

(i) $\odot$ This work is licensed under a Creative Commons Attribution-NonCommercial-NoDerivatives 4.0 International (CC BY-NC-ND 4.0) License. 